\title{
我国准分子激光技术发展现状及趋势研究
}

\author{
范元媛 ${ }^{1}$, 周朗 $^{1}$, 郭馨 $^{1}$, 王倩 ${ }^{1}$, 金春水 $^{2}$, 楼祺洪 $^{3}$ \\ (1. 中国科学院微电子研究所, 北京 $100029 ; 2$. 中国科学院长春光学精密机械与物理研究所, 长春 130033 ;
}

3. 中国科学院上海光学精密机械研究所, 上海 201800)

\begin{abstract}
摘要: 准分子激光具有波长短、能量大等特点, 在集成电路光刻、材料加工、医学、科研等领域具有重要的应用。与国外先 进水平相比, 我国高端准分子激光技术仍存在较大差距。本文首先简要介绍了准分子激光的特点和发展历史, 然后分析了国 内外准分子激光技术及相关典型应用的发展现状及需求，之后提出了我国准分子激光技术发展的主要问题。最后，针对相关 问题及需求, 建议未来重点在基础共性技术研究 (高性能高端深紫外波段元器件设计、制备与性能表征研究, 放电动力学等 基础理论及验证研究等）、长脉冲、高重频、大能量 / 功率技术、新兴或潜在应用领域及衍生技术等研究方面继续加大加深 研究力度, 为我国准分子激光的自主可控发展奠定基础。
\end{abstract}

关键词: 激光器; 准分子激光技术; 材料加工

中图分类号：TN248 文献标识码：A

\section{Development Status and Trend of China's Excimer Laser Technology}

\author{
Fan Yuanyuan ${ }^{1}$, Zhou Yi ${ }^{1}$, Guo Xin ${ }^{1}$, Wang Qian ${ }^{1}$, Jin Chunshui ${ }^{2}$, Lou Qihong ${ }^{3}$ \\ (1. Institute of Microelectronics of the Chinese Academy of Sciences, Beijing 100029, China; 2. Changchun Institute of Optics, \\ Fine Mechanics and Physics, Chinese Academy of Sciences, Changchun 130033, China; 3. Shanghai Institute of Optics and Fine \\ Mechanics, Chinese Academy of Sciences, Shanghai 201800, China)
}

\begin{abstract}
Excimer laser has important applications in integrated circuit lithography, materials processing, medicine, and scientific research due to its short wavelength and high pulse energy. However, there remains a big gap in the high-end excimer laser technology between China and the international advanced level. In this article, the characteristics and development history of excimer lasers were briefly introduced first. Second, the development status and requirements of the excimer laser technology and its related typical applications were analyzed in China and abroad. Third, the main issues of the development of domestic excimer laser technology were proposed. Finally, in view of the relevant problems and needs, it is suggested that in the future, more efforts should be made to deepen the research, including basic generic technology research (design, preparation, and characterization of high-performance and high-end deep UV components, basic theory and verification research of discharge dynamics, etc.); long pulse, high repetition rate, and high energy/power technology; as well as emerging or potential application fields and derivative technologies, so as to lay the foundation for the independent and controllable development of excimer lasers in China.
\end{abstract}

Keywords: laser; excimer laser technology; material processing

收稿日期 : 2020-03-26; 修回日期 : 2020-04-15

通讯作者: 周翊, 中国科学院微电子研究所研究员, 主要研究方向为激光技术及应用; E-mail: zhouyi1@ime.ac.cn 资助项目：中国工程院咨询项目“我国激光技术与应用 2035 发展战略研究” (2018-XZ-27)

本刊网址：www.engineering.org.cn/ch/journal/sscae 


\section{一、前言}

准分子是一种在激发态结合而基态离解的受激 二聚体。准分子激光的跃迁发生在束缚的激发态到 排斥的基态，属于束缚 - 自由跃迁 [1]。其特点是 基态不稳定，一般在振动弛豫时间内便分解为自由 粒子，而其激发态以结合的形式出现并相对稳定， 以辐射的形式衰减, 因而准分子激光具有高增益的 特点 [2]。准分子激光器是紫外波段大能量激光光 源, 是一种辐射脉宽为几十纳秒的紫外气体激光器。 由于具有光子能量高、波长短、空间相干性低（不 易产生干涉条纹）等一系列优势，准分子激光是目 前最有效、适合大规模工业生产的深紫外光源, 在 集成电路光刻、医疗、材料加工、科研等领域具有 广泛的应用。

早在 1960 年, Houtermaus 就提出了以准分子 为工作介质实现激光振荡的建议。1970 年, Basov 等首次采用强流电子束激发液态氙气二聚体得到 $\mathrm{Xe}_{2}$ 准分子激光输出, 激光波长为 $172 \mathrm{~nm}[3]$ 。此 后 50 年来，准分子激光技术得到了迅速发展，先 后实现了 $\mathrm{Kr}_{2}(145.7 \mathrm{~nm}) 、 \mathrm{Ar}_{2}(126.1 \mathrm{~nm}) 、 \mathrm{XeO}$ $(235 \mathrm{~nm}) 、 \mathrm{KrO}(180 \mathrm{~nm}) 、 \operatorname{ArO}(150 \mathrm{~nm}) 、 \mathrm{XeBr}$ $(282 \mathrm{~nm}) 、 \mathrm{XeF}(351 \mathrm{~nm}) 、 \mathrm{KrF}(248 \mathrm{~nm}) 、 \operatorname{ArF}$ $(193 \mathrm{~nm}), \mathrm{XeCl}(308 \mathrm{~nm}), \mathrm{KrCl}(222 \mathrm{~nm}) 、 \mathrm{ArCl}$ (175 nm) 等激光辐射 [4]。特别地, 以预电离放电 泵浦 $\mathrm{ArF}(193 \mathrm{~nm}) 、 \mathrm{KrF}(248 \mathrm{~nm}) 、 \mathrm{XeCl}(308 \mathrm{~nm})$ 为代表的稀有气体卤化物准分子激光的各项技术得 到迅猛发展，实现了准分子激光的商业化并广泛 应用于科研、工业、医疗等相关领域 [5]。20 世纪 90 年代, 随着准分子激光引入半导体光刻生产领 域, 大量准分子激光进入工业生产线, 极大地推动 了高重频、窄线宽、长寿命、高稳定性准分子激光 技术的发展。与此同时，其他微结构加工和材料处 理等工业应用, 诸如液晶平板退火、微细结构加工 和表面处理等也促进了大功率准分子激光技术的蓬 勃发展。

在大规模集成电路生产领域，光刻机一直是超 大规模集成电路生产中最关键的设备，而高性能的 准分子激光光源是光刻机的核心部件之一，是实现 高水平光刻的关键技术之一，也是限制我国集成电 路发展的关键部件之一，更是推动光刻技术发展的 “源”动力 [6]。目前国际上仅有美国 Cymer 公司
（现已被荷兰阿斯麦尔（ASML）公司收购）与日本 Gigaphoton 公司两大光刻光源制造商，它们对我国 进行技术封锁，严重限制了我国集成电路制造装备 的发展，相关技术壁垒亟待攻克。

在材料加工领域，复合材料、陶瓷、金属、纳 米材料等新兴或升级材料的出现, 对加工质量本身 提出了更高的要求。为了完全满足市场对性能与良 率的需求, 急需进一步提高加工可控性, 避免或减 少热影响区、次表面损伤等加工缺陷。准分子激光 由于具有热影响小、空间分辨率高、效率高、无污 染、不产生次表面破坏层等特点 [7], 同时大多数 材料对紫外激光具有很高的吸收率 $[8,9]$, 成为相关 材料加工领域的理想光源之一。

\section{二、国内外准分子激光技术发展现状及需求 分析}

\section{（一）国外发展现状及需求分析}

对于准分子激光器, 国外有比较成熟的商用产 品, 主要生产厂家有：美国的 Coherent 公司（包 含收购的 Lambda Physik 和 Tui Laser)、GAM Laser 公司, 日本的 Gigaphoton 公司，荷兰的 ASML 公司 (Cymer) 和加拿大的 Lumonics 公司等。从目前准分 子激光器生产商的相关产品可以看出, 准分子激光 光源发展需求主要分为两类：针对光刻需求一一高 重频, 同时要求极窄的光谱及极高的稳定性; 针对 工业加工需求一大单脉冲能量, 高平均功率。

针对光刻应用需求，国际上主要有荷兰 ASML 公司（Cymer）和日本 Gigaphoton 公司提供相应的 准分子激光产品, 相应功率从 $10 \sim 100 \mathrm{~W}$ 、光谱线 宽从 $0.5 \sim 0.1 \mathrm{pm}$ 、重复频率从 $2 \sim 6 \mathrm{kHz}$ 。高重复频 率可以提高加工产率，窄线宽可以保证芯片图案的 精细度, 减小系统中色差影响, 因此, 高重频和窄 线宽是光刻用准分子激光光源发展不断追求的指 标。表 1 为 ASML 公司 (Cymer) ArF 准分子激光 产品的发展历程。

针对工业生产及科研应用需求, 主要有美国的 Coherent 公司提供相应的准分子激光光源。主要应 用领域包括：聚合物标记等打标应用、光纤光栅刻 写等材料加工、燃烧诊断等测量应用、激光退火等 表面处理、近视矫正等医疗应用。工业及科研用准 分子激光器一般要求具有较高的稳定性和光斑均匀 
表 1 ASML 公司 (Cymer) ArF 准分子激光产品的发展历程

\begin{tabular}{lccc}
\hline 时间/年 & 型号 & 重复频率/kHz & 能量/mJ \\
\hline 2001 & Nanolith7000 & 4 & 5 \\
2002 & XLA100 & 4 & 10 \\
2003 & XLA200 & 4 & 15 \\
2006 & XLA300 & 6 & 15 \\
2007 & XLR500i & 6 & 10 \\
2009 & XLR600ix & 6 & 15 \\
2014 & XLR660ix & 6 & 20 \\
2017 & XLA700ix & 6 & $10 \sim 15$ \\
2018 & XLA800ix & 6 & $15 \sim 20$ \\
\hline
\end{tabular}

性。以制造平板显示器 (FPD) 的激光退火工序应 用为例, 其使用的准分子激光能量稳定性一般要求 小于 $2 \%$ 。

在材料加工与表面处理方面, Insung 等 [10] 使 用激光诱导单晶碳化硅的固态相位分离, 实现了多 层石墨烯的制备; 日本京都大学研究者利用 $\mathrm{KrF}$ 准 分子激光实现了侧壁粗糙度的降低，提高了波导的 通光性能并提升了抗拉强度, 热影响小、修复力强、 质量高 [11]; 日本 Kobayashi 等 [12] 利用 $193 \mathrm{~nm}$ 激 光加工碳纤维增强复合材料 (CFRP), 加工热影响 区在目前报道中属于较高水平。由于对复合材料疲 劳强度影响最小，具有综合最优的加工质量及加 工效率，美国 Coherent 公司、日本 Gigaphoton 公 司 $[13,14]$ 都将 CFRP 加工作为准分子激光器的重要 应用进行相关光源、材料加工技术的研究。此类应 用对光源稳定性、光斑均匀性以及定位精度等都提 出了越来越高的要求。

\section{（二）我国发展现状及需求分析}

我国准分子激光技术的研究工作开始于 20 世 纪 70 年代 [15], 主要研究单位包括中国科学院上 海光学精密机械研究所、安徽光学精密机械研究所、 长春光学精密机械与物理研究所、天津大学等, 其 研究主要集中在 $\mathrm{XeCl}$ 和 $\mathrm{KrF}$ 准分子激光器。20世 纪 90 年代之后, 我国科研型准分子激光向实用化方 向发展, 上海光学精密机构研究所和安徽光学精密 机械研究所开发了一些激光器产品, 并出口到国外。

从 2009 年起，在国家科技重大专项（02 专项） 的支持下，我国准分子激光技术获得迅速发展，中 国科学院光电研究院、上海光学精密机构研究所、
长春光学精密机械与物理研究所、合肥物质科学研 究院、光电技术研究所、上海微电子装备有限公司、 华中科技大学等单位参与了相关项目研发工作。目 前, 已攻克了一系列高性能准分子激光核心关键技 术, 实现了高重频 $(\mathrm{kHz})$ 、大能量 $(\mathrm{mJ}$ 级)、窄线 宽（亚 $\mathrm{pm}$ ) 准分子激光的稳定运转, 研发出第一 代光刻用准分子系统原理样机，目前正在进行技术 提升和面向产品的开发, 并初步建立了我国自主的 知识产权体系。图 1 为目前统计的准分子激光技术 国内发明专利申请量与国外来华发明专利申请量对 比。可见, 在 2009 年之前, 国内申请和国外来华 申请量仅分别为 7 项和 3 项; 在 2009 年之后，截 至 2018 年 3 月, 相关数据已分别激增至 203 项和 106 项。国内准分子激光技术战略布局如图 2 所示, 主要分布在放电腔设计、流场设计、准分子激光电

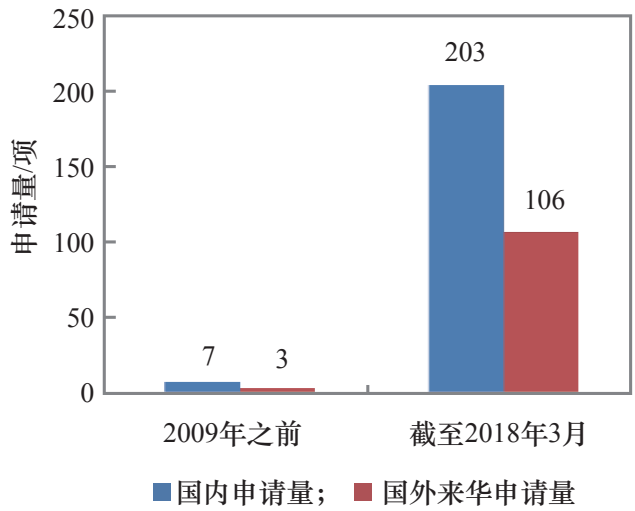

图 1 准分子激光技术国内发明专利申请量与 国外来华发明专利申请量对比图

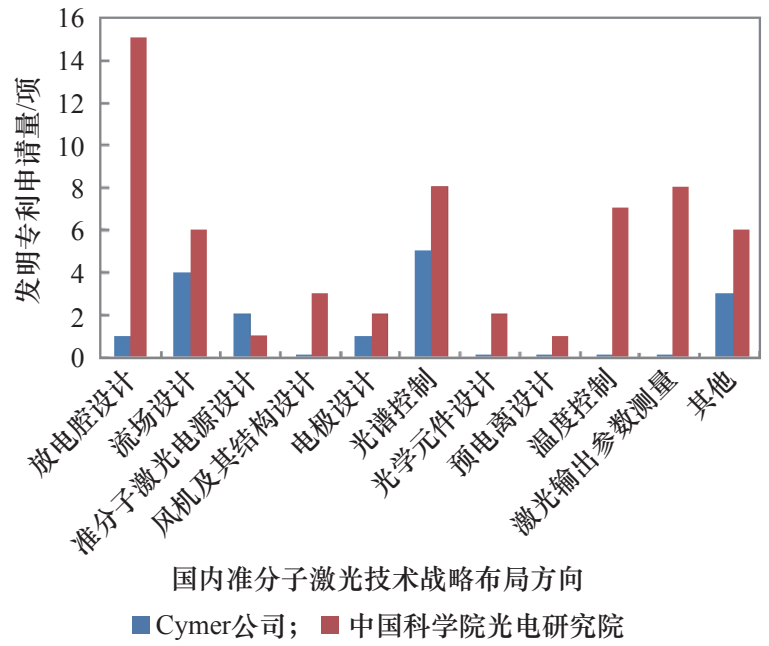

图 2 国内准分子激光技术战略布局 
源设计、电极设计、光谱控制、光学元件设计、预 电离设计等方面。

我国在准分子激光加工与处理方面的研究起步 于 20 世纪 80 年代 $[16,17]$ 。目前国内多家科研院所 及公司都在进行相关技术研发, 已取得一定的进展。 北京工业大学 [18] 加工 $\mathrm{SiC}$ 单晶的表面粗䊁度达 $4.11 \mathrm{~nm}$, 相比抛光前降低 $83 \%$; 上海交通大学 [19] 对激光诱导晶化氢化纳米硅薄膜进行了实验与模 拟, 研究明确了工艺过程与影响因素; 中国科学院 光电研究院 [20] 研究了 $\mathrm{SiC} 、 \mathrm{Al}_{2} \mathrm{O}_{3}$ 陶瓷的表面处 理并确定了烧蚀机制。天津大学、国防科技大学等 诸多科研机构也进行了相关的研究。

虽然我国在准分子激光研发方面已取得一系列 成果，但是，在基础性技术研究、新兴或潜在应用 领域及衍生技术等研究方面尚存在较大不足。

\section{三、我国准分子激光技术发展问题分析}

\section{（一）高性能紫外激光器用高端元器件短缺}

高性能的紫外激光光源对所使用的高端元器件 提出了越来越高的要求。以光学元件及薄膜元件为 例，在国家重大专项的资助下，国内制备的紫外光 学元件及薄膜元件的性能有了长足的进步, 所制备 的光学薄膜元件已可以较好地满足光学和光谱性能 方面的要求，在抗激光辐照损伤抑制和提高使用寿 命方面, 也取得了显著进展。然而, 前期的大量研 究结果也显示目前国内的光学元件及薄膜元件的综 合性能与高性能紫外激光器的实际和潜在要求之间 还存在一定的差距, 面向超高性能紫外激光器的应 用需求，光学元件及薄膜元件的长期抗激光辐照损 伤能力还有待进一步提高。造成这种差距的原因主 要包括: 首先, 光学元件涉及材料生长、表面加工 及应用等多个环节, 各环节都存在多种影响抗激光 辐照性能的因素，导致对其具体影响机理及规律的 研究难度很大; 其次, 国内在高端光学元件材料及 加工方面的前期基础薄弱, 也对后续的光学镀膜工 作及长期应用评价工作造成障碍；最后，到目前为 止, 国内还没有形成对高性能紫外激光器光学元件 及薄膜元件的综合检测和评价的有效技术和标准， 使得对光学元件的材料、加工、镀膜及应用等环节 中各影响因素的优化工作不能高效开展。

\section{（二）基础性研究薄弱}

目前我国在准分子激光研究方面主要是参照国 外成熟产品, 主要集中在依靠实验手段解决工程技 术问题，基础性技术积累相对薄弱。

以放电动力学为例, 准分子激光系统的运转过 程是高压气体放电等离子体激发光辐射过程, 对放 电过程特性的研究是系统设计的核心和根源。放电 过程特性的准确分析, 对系统的优化改善都将具有 极其重要的作用。通过仿真, 研究准分子激光系统 的放电动力学特性, 实时预测工作气体的成分变化, 深化理解从工作气体电离到激光辐射的物理过程, 明确关键因素, 可对准分子激光系统的结构特性优 化提供设计指导，提高系统运转相关实验验证的准 确性和可靠性。目前, 我国在放电动力学等基础性 研究方面处于刚刚起步状态, 难以指导新技术、新 产品的开发。

\section{（三）高端准分子激光技术与国外领先水平尚存在} 较大差距

在超大规模集成电路光刻和超精细加工等当前 应用及未来潜在应用中, 要求紫外激光的输出指标 越来越苛刻, 主要包括: 要求紫外激光的输出脉冲 能量、功率和光谱性能指标越来越高, 同时要求其 输出脉冲能量和光谱性能具有极高的稳定性与可控 变化特性。

目前, 我国虽然在准分子激光技术及研发方面 取得了一系列突破性进展, 但和国外领先水平相比, 尚存在着较大的差距, 造成这种差距的原因主要包 括：国内基础薄弱，人才队伍（特别是高端领军人 才）短缺，技术及产品落后。

\section{四、我国准分子激光技术未来发展方向建议}

在国家科技重大专项的支持下，我国准分子 激光技术获得了迅速发展, 攻克了一系列高性能 准分子激光核心关键技术。然而, 和国外领先水 平相比, 尚存在着较大的差距, 仍需国家层面的 大力支持。

针对于当前我国准分子激光技术发展过程中的 问题以及相关技术短板及需求, 建议未来重点发展 方向主要包括以下几点。 


\section{（一）基础共性技术研究}

1. 高性能准分子激光器用高端元器件设计、制 备与性能表征研究

以光学元件及光学薄膜的制备及其长期性能稳 定性测试与评价为例, 在准分子激光光学系统中, 由于紫外激光单光子能量高, 高能光子与物质相互 作用导致光学元件更易产生激光损坏和性能退化, 极大影响紫外激光光学系统的通光能力、性能、稳 定性和寿命。因此, 高性能光学元件及光学薄膜的 制备极其重要, 需要开展材料严格䇥选、光学表面 超精密加工工艺优化、高性能薄膜制备工艺优化, 以及应用环境对抗辐照性能影响评价等一系列工作。

另外, 当前针对紫外激光器光学元件及薄膜元 件的性能评价还主要依赖于一些较常规的技术和手 段, 远远无法满足高性能光学元件及薄膜元件制备 的需要。因此, 有必要在现有测试和评价技术的基 础上, 针对紫外激光器光学元件及薄膜元件抗激光 辐射能力和长期性能稳定性的要求, 完善建立基于 激光损伤阈值组合测试的短期评价方法和基于低能 激光辐照组合的长期评价方法相结合的高效系统性 评价方案, 以此作为高性能 $\mathrm{CaF}_{2}$ 等光学材料篎选、 光学表面超精密加工工艺优化、高性能薄膜制备工 艺优化、以及应用环境影响研究开展所需的新的技 术支撑。

\section{2. 基础性理论及验证研究}

从基础理论模拟仿真、基础原材料特性、基础 元器件性能特性分析等方面, 加强基础性研究以及 验证工作。以放电动力学为例, 对放电动力学特性 研究主要考察研究准分子激光器高压气体放电等离 子体激发光辐射过程, 其中涉及气体混合配比情况、 腔内气体压力情况、主电极的设计、预电离电极的 设计、放电电极间距、放电电压情况、电极预电离 机制结构等多方面的内容, 进行的最优化设计, 从 而可以有效辅助新技术及产品开发, 提高新产品开 发效率, 节约开发成本。

\section{（二）长脉冲、高重频、大能量 / 功率技术研究}

紫外激光由于波长短, 单光子能量大, 在加工 等领域具有优势的同时, 也带来光学元件易受到损 伤等难题, 增加激光脉宽可以有效地解决这一问题。 面向应用需求, 长脉冲紫外激光技术是重要研究方 向之一, 但常规激光器脉宽受限于激光上能级寿命、
泵浦电源等因素，更宽的激光脉宽较难实现。

激光重频直接影响加工产率, 二者呈正比例关 系。目前国际上光刻用准分子激光光源最高重频是 $6 \mathrm{kHz}$, 若要在其他条件相同的情况下继续提高加 工产率, 势必要继续提高激光重频, 而为了提升激 光重频, 必须解决泵浦电源、光学材料、驱动风机、 流场、激波、散热等一系列问题。针对应用的前瞻 需求, 有必要开展更高重频激光光源研究, 从而实 现我国在这一技术领域的领先地位, 为更高性能激 光光源的开发奠定基础。

在智能手机、平板显示器等领域广泛应用的新 型显示器, 包括有源矩阵液晶显示器 (AMLCD) 和有源矩阵有机发光二极管 (AMOLED) 显示器等 是下一代电子产品的核心之一，广泛应用于日常电 子消费类产品和不同工业领域，具有巨大的市场价 值, 是国家经济建设和社会发展的重要领域。其中, 低温多晶硅（LTPS）的制备是最关键的加工工艺之 一，而准分子激光退火已经成为当下屏制造中制备 有效的多晶硅层的首选方法。该光源一般要求单脉 冲能量至少数百毫焦, 因此, 有必要开展大能量 / 功率技术研究, 这将为我国在该领域开发具有核心 自主知识产权的装备奠定基础。

\section{（三）新兴或潜在应用领域及衍生技术研究}

在现有研究成果的基础上, 针对光电对抗、高 精度光学系统检测等应用, 开展电磁干扰、辐射标 定、综合参数诊断、光谱调谐控制等进行技术辐射 转移。

针对新兴或潜在的应用需求, 如微加工制造(包 括：多层石墨烯材料的制备; CFRP 等复合材料的 切割、微孔、表面羟基化; $\mathrm{SiC}$ 等脆硬陶瓷材料的 抛光、改性; 纳米材料的诱导晶化、沉积; 生物材 料的选择性切断、微流控芯片等器件的微加工等)、 高精度 / 性能元器件表面处理等应用领域, 有针对 地开展相适应的准分子激光技术及相关性能优化研 究, 为最终带动新的应用领域发展奠定基础。

\footnotetext{
参考文献

[1] 楼祺洪, 徐捷, 傅淑芬, 等. 脉冲放电气体激光器 [M]. 北京: 科 学出版社, 1993.

Lou Q H, Xu J, Fu S F, et al. Gas laser with pulsed discharge [M] Beijing: China Science Publishing \& Media Ltd., 1993.

[2] Colin W. History of gas lasers, Part 2: Pulsed gas lasers [J]. Optics
} 
\& Photonics, 2010, 21(2): 20-27.

[3] Basov N G, Danilychev V A, Popov Y M, et al. Laser operating in the vacuum region of the spectrum by excitation of liquid xenon with an electron beam [J]. JETP Letters, 1970, 12(10): 329.

[4] Rokitski R, Ishihara T, Rao R, et al. High reliability ArF light source for double patterning immersion lithography [J]. Optical Microlithography XXIII, 2010, 7640: 1-7.

[5] 余吟山, 游利兵, 梁勋, 等. 准分子激光技术发展 [J]. 中国激光, 2010, 37(9): 2253-2270.

Yu Y S, You L B, Liang X, et al. Progress of excimer lasers technology [J]. Chinese Journal of Laser, 2010, 37(9): 2253-2270.

[6] 楼祺洪, 袁志军, 张海波. 光刻技术的历史与现状 [J]. 科学, 2017, 69(3): 32-36.

Lou Q H, Yuan Z J, Zhang H B. History and status of lithography [J]. Science, 2017, 69(3): 32-36.

[7] 何立文, 方晓东. 准分子激光微加工应用研究进展 [J]. 量子电 子学报, 2018, 35(6): 641-648.

He L W, Fang X D. Progress of application of excimer laser micromachining $[\mathrm{J}]$. Chinese Journal of Quantum Electronics. 2018, 35(6): 641-648.

[8] Wang H, Lin H, Wang C, et al. Laser drilling of structural ceramicsA review [J]. Journal of the European Ceramic Society, 2017, 37(4): 1157-1173.

[9] 赵读亮. 工业及医用准分子激光系统关键技术研究 [D]. 合肥: 中国科学技术大学(博士学位论文), 2017.

Zhao D L. Study on key techniques of medical and industrial excimer laser system [D]. Hefei: University of Science and Technology of China (Doctoral dissertation), 2017.

[10] Insung C, Hu Y J, Hyeyoung S, et al. Laser-induced phase separation of silicon carbide [J]. Nature Communications, 2016, 7: $1-14$.

[11] Mitwally M E, Tsuchiya T, Tabata O, et al. Improvement of tensile strength of freestanding single crystal silicon microstructures using localized harsh laser treatment [J]. Japanese Journal of Applied Physics, 2014, 53(6S): 1-6.

[12] Kobayashi M, Kakizaki K, Oizumi H, et al. DUV high power lasers processing for glass and CFRP [C]. Prague: SPIE Optics + Optoelectronics, 2017.

[13] Yuji S, Masahiro T, Tatsuya N, et al. Experimental study of CFRP cutting with nanosecond lasers [J]. Transactions of JWRI, 2013, 42(1): 23-26.

[14] Delmdahl R, Gabler F. Excimer lasers clean CFRPs for optimized adhesive bonding [J]. The Laser User, 2013 (72): 10.

[15] 楼祺洪. 准分子激光器的发展和应用 [J]. 中国激光, 1994, A21(5): 361-364.

Lou Q H. Progress of excimer lasers and its applications [J]. Chinese Journal of Lasers, 1994, A21(5): 361-364.

[16] 邱佩华. 准分子激光器的技术应用走向商品化 $[\mathrm{J}]$. 激光与光电 子学进展, 1987 (5): 25-27.

Qiu P H. Commercialization of excimer laser technology application [J]. Laser \& Optoelectronics Progress, 1987 (5): 25 27

[17] 袁才来, 上官诚, 叶超, 等. 用193毫微米 $\operatorname{ArF}$ 准分子激光对 $\mathrm{HgBr} \_2$ 进行单光子和双光子解离的研究 [J]. 激光, 1981 (4) 62-63.

Yuan C L, Shangguan C, Ye C, et al. Study on single-photon and two-photon dissociation of $\mathrm{HgBr} 2$ by $193 \mathrm{~nm} \mathrm{ArF} \mathrm{excimer} \mathrm{laser}$ [J]. Laser, 1981 (4): 62-63.

[18] 李政达. $6 \mathrm{H}-\mathrm{SiC}$ 单晶与 $\mathrm{YBCO}$ 超导薄膜激光抛光机理与工艺研 究 [D]. 北京: 北京工业大学(博士学位论文), 2017

Li Z D. Study on laser polishing mechanism and technology of $6 \mathrm{H}$ silicon carbide monocrystal and YBCO superconducting film [D] Beijing: Beijing University of Technology (Doctoral dissertation), 2017.

[19] 邓黎杰. 氢化纳米硅薄膜准分子激光诱导晶化的研究 [D]. 上 海: 上海交通大学(博士学位论文), 2015.

Deng L J. The study on excimer laser crystallization of nanocrystalline silicon thin films [D]. Shanghai: Shanghai Jiao Tong University (Doctoral dissertation), 2015.

[20] Guo X, Ding J B, Zhou Y, et al. Ablation effects and mechanism of sintered silicon carbide ceramics by ArF excimer laser [J]. Chinese Optics Letters, 2018, 16(9): 59-63. 